\title{
Multifrequency Driven Nematics
}

\author{
Noureddine Bennis *, Jakub Herman $₫$, Aleksandra Kalbarczyk, Przemysław Kula \\ and Leszek R. Jaroszewicz
}

Faculty of Advanced Technologies and Chemistry, Military University of Technology, 2 gen. S. Kaliskiego St., 00-908 Warsaw, Poland; jakub.herman@wat.edu.pl (J.H.); aleksandra.kalbarczyk@wat.edu.pl (A.K.); przemyslaw.kula@wat.edu.pl (P.K.); leszek.jaroszewicz@wat.edu.pl (L.R.J.)

* Correspondence: noureddine.bennis@wat.edu.pl

Received: 10 May 2019; Accepted: 23 May 2019; Published: 27 May 2019

\begin{abstract}
Liquid crystals act on the amplitude and the phase of a wave front under applied electric fields. Ordinary LCs are known as field induced birefringence, thus both phase and amplitude modulation strongly depend on the voltage controllable molecular tilt. In this work we present electrooptical properties of novel liquid crystal (LC) mixture with frequency tunable capabilities from $100 \mathrm{~Hz}$ to $10 \mathrm{KHz}$ at constant applied voltage. The frequency tunability of presented mixtures shown here came from composition of three different families of rodlike liquid crystals. Dielectric measurements are reported for the compounds constituting frequency-controlled birefringence liquid crystal. Characterization protocols allowing the optimum classification of different components of this mixture, paying attention to all relevant parameters such as anisotropic polarizability, dielectric anisotropy, and dipole moment are presented.
\end{abstract}

Keywords: nematic; dual frequency nematic; dielectric anisotropy; optical modulation

\section{Introduction}

Spatial light modulators (SLMs) based on liquid crystals (LCs) require very specific combinations of physical properties of liquid crystal mixtures to operate efficiently, requiring different combinations of components. Multicomponents mixtures of LCs find widespread use in photonic applications. The reason for this, is the wide temperature range necessary for applications [1]. The more important physical properties of LCs mixtures include electric permittivities, optical refractive indices, elastic constants, and viscosities. In order to understand the dielectric properties of LCs mixtures, some knowledge of the behavior of each component of the mixture is necessary. Owing to their electrical and optical properties, they are very sensitive to external electric and magnetic fields and they play an important role in many types of photonic devices enabling the development of multiphase spatial light modulators (SLMs) that can perform high-resolution, dynamic, optical beam positioning as well as temporal and spatial beam shaping. The optical system of such as spatial light modulators can perform with extremely high-resolution having a very small pixel size of about a few $\mu \mathrm{m}$. This small resolution makes the latest generation of microdisplays based on LCs useful for many applications. However, we shall be concerned with negative physical effects related to smaller pixel sizes because of the inherent elasticity of the liquid crystal (LC) material, the surface anchoring of the alignment layer, and the fringing field of the discrete electrodes' voltage distribution [2]. The electrical field that exists between a pixel turned on and its neighbor at a different voltage causes part of the liquid crystal molecules to adopt a tilt angle opposite to the one in the main part of the pixel [3]. In order to avoid the fringing field effect originating from different values of voltage distribution, a digital driving frequency such as a pulse width modulation is needed. In this case, equal voltage with a predetermined frequency should be applied to adjacent pixel electrodes. As well as the amplitude of applied voltage 
could alter the reorientation of LC molecules, also its frequency may alter their response. Thus, we need to understand the most important information about the molecular dynamics of LCs in the presence of the alternating electric field. When a highly anisotropic molecules of LCs are found in an electric field their electric polarization depends on their orientation with respect to it. The frequency dependence can be described from the physical origin of the induced polarization of the molecules. This induced polarization interacts with an external electric field and the torque tries to align the long molecular axis parallel or perpendicular to the external electric field depending of the sign of the dielectric anisotropy. If the applied field varies with time, then the frequency dependence of the permittivity is an additional property of the LCs material under study. Thus at much higher frequency of the applied electric field, the molecular dipoles do not rotate fast enough to contribute to the dielectric response, therefore the phase shift between the electric field $\mathrm{E}$ and electric polarization occurs. This delay yields to the dielectric relaxation of the LC molecules, causing the dielectric dispersion and losses [4]. For the uniaxial nematic LCs there are two independent components of the electric permittivity, parallel $\varepsilon_{\|}$and perpendicular $\varepsilon_{\perp}$ to the axis of symmetry. Both principal components of the dielectric permittivity tensor show different frequency dependencies and temperature dependencies as well [5-7]. Perpendicular component $\varepsilon_{\perp}$ exhibits the high frequency process which can be treated as an ordinary Debye dispersion of fluid systems which usually occurs in the microwave region. However, $\varepsilon_{\|}$may exhibit in some cases an additional dispersion at much lower frequencies in the radio-frequency region. This low-frequency dispersion of $\varepsilon_{\|}$is caused by orientational relaxation of the permanent dipole moment of the parallel component to the long molecular axis. Maier and Meier are the first who formulated these ideas more qualitatively by applying Onsager's theory of static polarization to nematic LCs [8]. Measurements of the dielectric properties of LCs give relaxation frequencies, which reflect dynamic processes involving a change in electric polarization that results from the chemical structure of the mesogen forming the LC material. Many mixtures of LCs exhibit a single dielectric relaxation even if the components individually have relaxations in different frequency ranges. However, in some mixtures of mesogens having significantly different structures, for example mixtures of two-ring and three-ring mesogens, two separated relaxations are sometimes detected [9]. The possibility of designing mixtures with very low relaxation frequencies is useful for a dual frequency addressing displays [10]. Such mixture is usually formed by a combination of many components such as molecules having large transverse dipole moment with $\Delta \varepsilon<0$ and molecules with a large longitudinal dipole moment with $\Delta \varepsilon>0$ [11]. The availability of a dielectric anisotropy of either sign depending on the frequency of the applied field allows the possibility to improve the dynamic response of electro-optical devices. One of the main drawbacks of Dual frequency liquid crystal (DFLC) was the high crossover frequencies; problems arising when high frequencies are applied $(>50 \mathrm{kHz})$ are higher cost in the driving circuit, larger heat dissipation, and stronger dielectric heating [12]. For this reason, the research on materials with low crossover frequencies led to novel materials [13-15]. In this work, we report on LCs mixture design by mixing three different families of LC compounds. Two groups of the components are dielectrically positive nematics, having different generated dipole moment, and one group of compounds is dielectrically neutral, which has been chosen as viscosity adjusting component. The resulting mixture meets the requirements of the large positive dielectric anisotropy LCs to be continuously controlled by low frequencies of the electrical applied voltage. The dielectric anisotropy of the proposed mixture goes to zero instead of being negative at high frequencies of the electric field. The mesomorphic properties of the three components of the mixture under study and electro-optical features in new formulated mixture are reported.

\section{Influence of NLC Mixtures on Dielectric Relaxation}

Particular semiempirical models have been regularly used in the literature to allow the prediction of the electrooptical parameters such as the dielectric anisotropy $\Delta \varepsilon$ and the birefringence $\Delta \mathrm{n}$ of dielectric materials. These two parameters can be derived from single molecule parameters, such as the dipole moment $\mu$ and the polarizability $\alpha$, using the Maier-Meier theory [8]. According to this 
theory, the supramolecular parameter $\Delta \varepsilon$ can be connected to the principal component of the molecular polarizability and the value and angular position of permanent electric dipole moment $\mu$.

Dielectric relaxation in LC material is influenced by the molecular structure used in the liquid crystal mixture and also by other factors such as intermolecular interactions and the local viscosity and temperature. This dependence gives raise to the possibility of designing liquid crystal mixtures with very low relaxation frequencies of electric permittivity parallel $\varepsilon_{\|}$, which is useful for many applications [16]. Apart from traditional SLM, the unique properties of this LC mixture can be used in all kind of optical phase modulators recently reported, e.g., adaptive lenses [17], beam steering [18], correction of aberrations [19], 3D vision applications [19-22], novel aberrations correctors for rectangular apertures [23], microaxicon arrays [24], multioptical elements [25], hole-patterned microlenses [26], multifocal microlenses [27], high fill-factor microlenses [28], frequency controlled [29] microlenses, optical vortices [30], lensacons, and logarithmic axicons [31]. The LC mixtures used in this work have been designed by mixing three different families of LC compounds to meet the requirements of the LC to be controlled by low frequencies of the electrical applied voltage. We have formulated the mixture using the set of compounds belonging to three different groups (see Table 1).

Table 1. General molecular structures of compounds used to form the investigated nematic mixture and their weight $\%$.

Components Chemical Structure Weight \%

The investigated material is a nematic composition of three different families of rod like LCs. The chemical formulas and the bond dipole moments of different groups forming the multifrequency driven nematic are shown in Figure 1. These structures belong to fluorine substituted 4-[(4-cyanophenoxy)carbonyl]phenyl 4-alkylbenzoates [32] (component I), fluorine substituted alkyl-alkyl phenyl-tolanes [32-34] (component II), and alkyl-alkyl bistolanes [35,36] (component III). Each component is characterized by their strong nematogenic character.

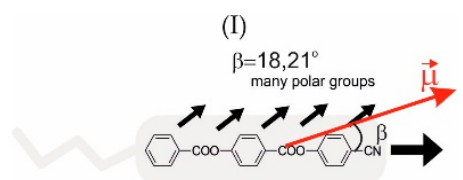

(a)

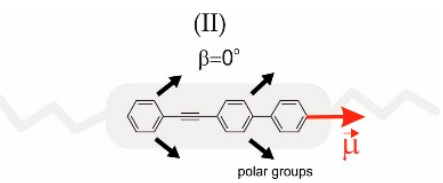

(b)

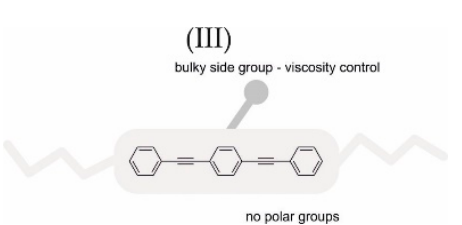

(c)

Figure 1. The structures of the three different groups forming the mixture: (a) component I, (b) component II and (c) component III. Red arrows indicate the position of the molecular dipole moments.

Two of the components are dielectrically positive nematics and one is dielectrically neutral. Components I and II have a large longitudinal dipole moment. Group I structures (Figure 1a) show a strong dipole moment that can be estimated as a vector sum of the single moments of all intramolecular chemical bonds. Their positions and orientations within the molecule for these dipole moments are 
additives, having substantial influence on nematic ordering. The largest contribution to the dipole moment of component I comes from the cyano group. This in turn is coupled with two ester groups that additionally enhance dipole moment value. The longitudinal electric dipole moment of the component (I) is about 12,4 debye due to a triple $-\mathrm{CN}$ bond; the permanent dipole moment forms an angle with the molecular axis equal to $18.21^{\circ}$. This group of compounds is characterized by dielectric anisotropy $\Delta \varepsilon$ in the range from 60 to 80 , which can be realized by the use of polar chemical groups, making this component a highly polar nematics liquid crystal. Additionally component I shows moderate molecular anisotropy and moderate electronic polarizability [36]. These highly polar LCs with high positive dielectric anisotropies tend to operate at lower voltages. Medium birefringent component II used in this mixture has a dipole moment of about 3 debye and $\Delta \varepsilon$ is in the range from +2 to +4 at $1 \mathrm{kHz}$. It has low melting enthalpies in order to enhance an excellent solubility with other two groups of components of the mixture. The group III is mainly used as diluters with which one may maintain the proper viscosity of LCs mixtures [32-34]. This component does not consist of polar substituents nor polar groups, therefore is a nonpolar highly birefringent liquid crystal. Bistolanes III show high anisotropy of molecular polarizability, as a result of a strongly conjugated system of benzene rings and carbon-carbon triple bonds [37]. In this component there is only an induced polarization that consists of two parts: the electronic polarization and the ionic polarization. Its dipole moment is $\mu=0$. Thus, its $\Delta \varepsilon$ is expected to be small $(\Delta \varepsilon=+0,7$ at $1 \mathrm{kHz})$ and is considered as medium to high birefringent with high nematogenity. This component is laterally substituted with short alkyl chains (methyl, ethyl, trifluoromethyl), shows strong nematic character, and therefore it minimizes the smectic formation in multicomponent systems under study. In contrast, in the LCs with polar molecules groups (I) and (II), there is, in addition to the total induced polarization, the orientation polarization due to the tendency of the permanent dipole moments to orient themselves parallel to the electric field.

\section{Dielectric Measurements Results}

In the context of the dielectric properties of the three components forming the multifrequency driven LCs mixture, their dielectric properties are of interest from a number of aspects. In this section we will focus on their dielectric relaxation spectra. the characterization involves the measure of the electric permittivity tensor over a range of frequencies from $100 \mathrm{~Hz}$ to $1 \mathrm{MHz}$. Dielectric investigation was carried out on the three components forming the mixture in planar aligned cells with thicknesses of $0.7 \mathrm{~mm}$. The alignment was induced on the substrates by spin-coating polyimide SE-130 (Nissan Chemical Industries, Ltd., Tokyo, state, Japan), then baking at $180^{\circ} \mathrm{C}$ for $1 \mathrm{~h}$. The coated substrates were rubbed in the same direction and were assembled in antiparallel orientation. For this measurement, an external magnetic field was applied to the cell in order to orient the LCs molecules. In this case, two experimental geometries were applied; the parallel and perpendicular orientations of the director with respect to the electric field have been achieved by turning the electromagnet by $90^{\circ}$ enabling the measurement of the $\varepsilon_{\|}$and $\varepsilon_{\perp}$ which are parallel and perpendicular permittivity tensor components, respectively. The frequency dependences of the real (upper graphs) and imaginary (lower graphs) parts of the electric permittivity measured for the nematic phase of the three groups are shown in Figure 2.

The component (I) has relatively long molecules, therefore the resulting mesophases tend to be of high viscosity and are difficult to align; it has high melting and clearing temperatures and accordingly has very high viscosity which makes difficult the characterization of their dielectric properties. Therefore, the dielectric properties of this compound have to be measured in other neutral nematic matrices in order to extrapolate their resulting dielectric properties. In Figure 2a, only $10 \%$ molar concentration of component (I) has been used. The dielectric results of the pure material would have about 10 times higher $\Delta \varepsilon, 10$ times higher $\varepsilon_{\|}$, and more than 10 times lower relaxation frequency. For this component, the relaxation processes are detected at low frequencies and are more characteristic of collective modes normally associated with orientational relaxation of the permanent dipole moments. Components (II) and (III) have already much lower crystalline nematic transition temperatures, and therefore, they could be evaluated as pure systems. Those materials were 
tested at $51{ }^{\circ} \mathrm{C}$ and $58{ }^{\circ} \mathrm{C}$ respectively. Under given temperatures, these components are far from nematic-isotropic transition, in consequence their properties within the nematic phase are flattened. The dielectric permittivity constants $\varepsilon_{\|}$and $\varepsilon_{\perp}$ of the component III do not depend on frequency, therefore, dielectric losses are very small in the frequency range $[100 \mathrm{~Hz}, 15 \mathrm{KHz}]$. Figure 2 shows that the constituents of the formulated mixture have different dielectric responses to an external electric field of variable frequency. Note that the frequency dependence of the parallel component of electric permittivity is different for each group. We have formulated the mixture using the set of compounds belonging to three different families (See Table 2).
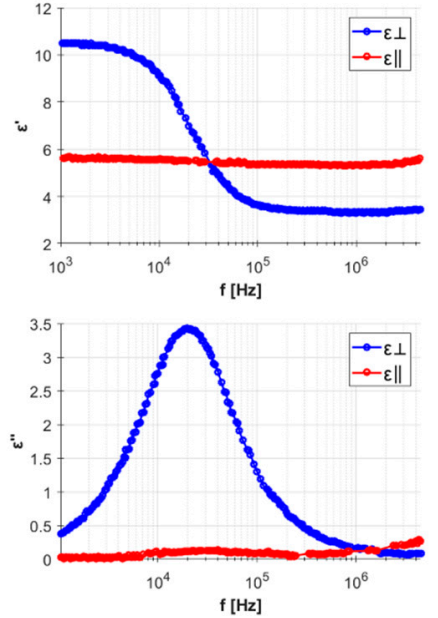

(a)
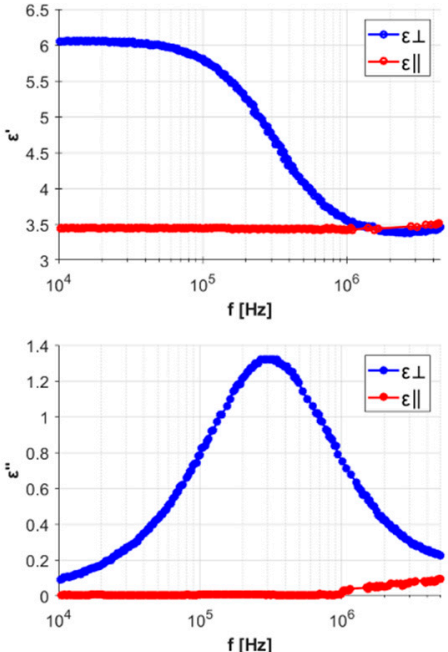

(b)
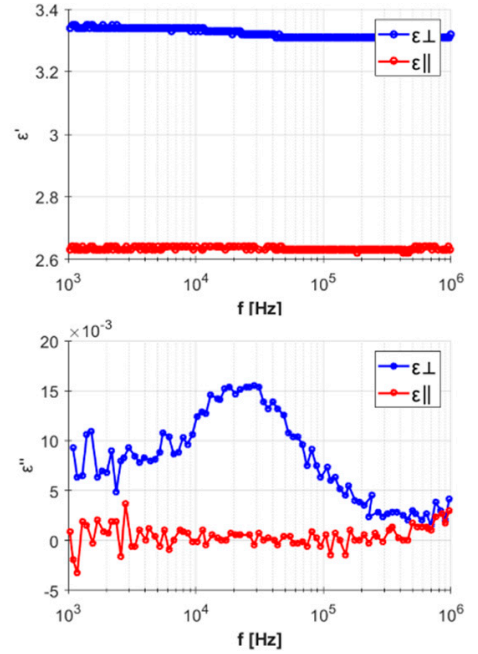

(c)

Figure 2. Frequency dependence of the real part (upper) and imaginary part (lower) of the complex permittivity of the formulated mixture in the measuring frequency range $100 \mathrm{~Hz}$ to $1 \mathrm{MHz}$ for the three different components of the mixture: (a) component (I), (b) component (II) and (c) component (III).

Table 2. Mesomorphic properties of the three components of the mixture under study.

\begin{tabular}{cccccc}
\hline Group & Polarity & $\begin{array}{c}\text { Anisotropic } \\
\text { Polarizability }\end{array}$ & $\begin{array}{c}\text { Dipole } \\
\text { Moment }\end{array}$ & $\begin{array}{c}\text { Dielectric } \\
\text { Anisotropy @ 1KHz }\end{array}$ & Viscosity \\
\hline I & high & moderate & $12,4 \mathrm{D}$ & $(60-80)$ & very high \\
II & moderate & high & 3D & $(2-4)$ & low \\
III & very low & high & $0 \mathrm{D}$ & 0,7 & moderate \\
\hline
\end{tabular}

Figure 3 shows the frequency dependence of the real and imaginary part of the complex permittivity of the formulated mixture in the measuring frequency range $100 \mathrm{~Hz}$ to $1 \mathrm{MHz}$. These results show that the mixing up of the three groups results in a positive nematic mixture with strong dependence on the frequency of the applied electric field. At a low frequency regime, the nematic mixture exhibits a large positive dielectric permittivity of about 9.5 and above $100 \mathrm{~Hz}, \varepsilon_{\|}$starts to decrease linearly as frequency increases. However, $\varepsilon_{\perp}$ is kept constant at a value of 3.5. In the frequency range $100 \mathrm{~Hz}$ to $1 \mathrm{MHz}$, both $\varepsilon_{\|}$and $\varepsilon_{\perp}$ stay at about 3,5. As a result, a continuously varying dielectric anisotropy, from 6 to 0 in the frequency range $100 \mathrm{~Hz}-15 \mathrm{KHz}$, is obtained. This mixture could be employed in optical devices which can readily be used as multiphase spatial light modulators controlled by a frequency.

The dielectric spectra showed in Figure $3 a$ can be presented in the form of a Cole-Cole plot. This diagram represents a series of two interlinked semicircles whose radius of the semicircle are inversely proportional to the relaxation time, indicating two relaxation mechanisms separated in frequency. These results may then be explained as different dynamic modes associated with relaxation of different dipoles group, each relaxing at a different frequency. Assuming that, these contributions to the electric permittivity occur at sufficiently different frequencies, and therefore, they can be separated 
in the dielectric spectrum. The dashed lines in Figure $3 \mathrm{c}$ mark two Debye-type processes fitted to the spectra. From those results, it seems that for our mixture two relaxation processes appear in the Cole-Cole plot corresponding to frequencies $3 \mathrm{KHz}$ and $34 \mathrm{KHz}$, which show evidence of two or more separate relaxation processes.

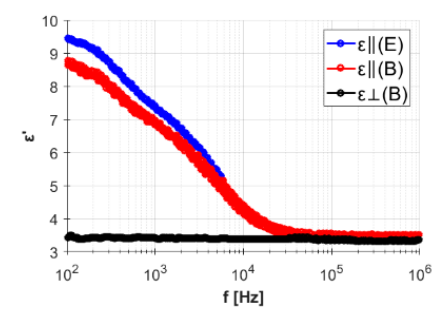

(a)

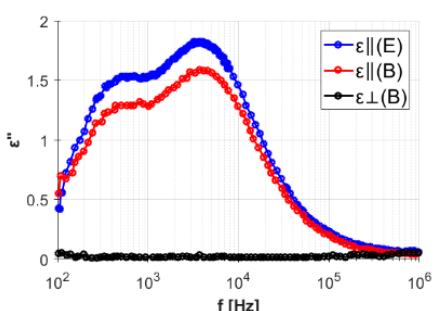

(b)

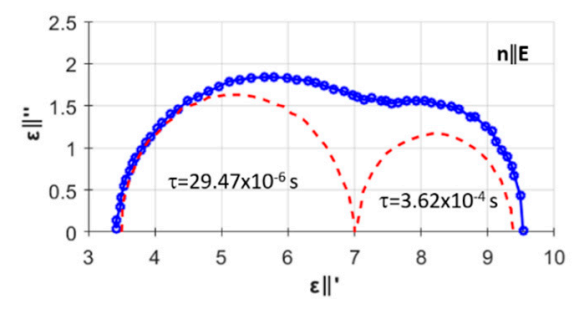

(c)

Figure 3. Frequency dependence of (a) real and (b) imaginary parts of the complex permittivity plotted in the measuring frequency range $100 \mathrm{~Hz}$ to $1 \mathrm{MHz}$. (c) Cole-Cole plot showing relaxation process with two time constants.

Figure 4 shows the results of the optical retardance versus the alternative voltage waveform applied. For optical retardance measurements, we have used cells prepared with the same alignment method described above. However, the thickness of the assembled cells has been reduced $5 \mu \mathrm{m}$ and next filled by the formulated mixture (Figure 4a) and by conventional liquid crystal 6CHBT (Figure 4b) when the voltage varies from $0 \mathrm{~V}$ to $15 \mathrm{~V}$ at a constant frequency. A maximal phase modulation depth obtained at $\lambda=6323 \mathrm{~nm}$ is $5 \pi$ in case of 5005 and $2.7 \pi$ in case of 6CHBT. Upon changing the frequency of the applied signal, a change in the retardance modulation is observed in the formulated mixture. This change is determined by the tunability of dielectric anisotropy by the frequency of the applied electric field. However, for LCs 6CHBT, since the relaxation frequency occurs in the $100 \mathrm{MHz}$ region [7] no dependence of optical retardation on the frequency has been detected.

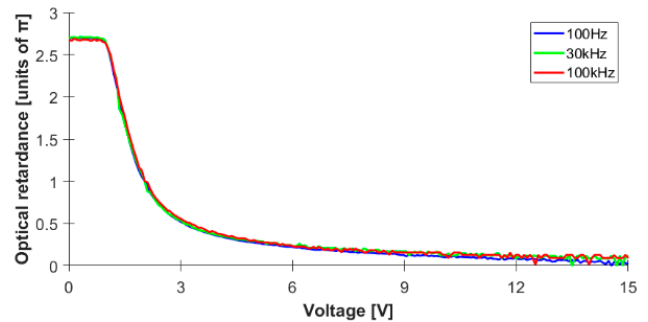

(a)

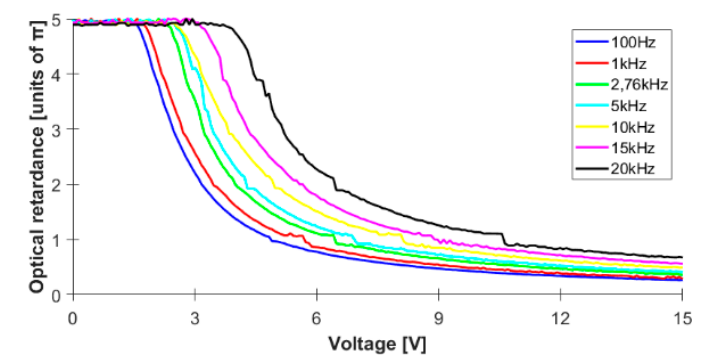

(b)

Figure 4. Voltage-dependent phase changes at different frequencies measured in $5 \mu \mathrm{m}$ cell filled with: (a) formulated mixture, (b) 6CHBT liquid crystals.

These results show that as the frequency increases the electro-optical characteristics of the cell filled with multifrequency driven nematics shifts toward the higher operation voltage. The frequency dependence of the threshold voltage shown in Figure 4a reflects the frequency dependence of the dielectric anisotropy of the proposed LCs material. This frequency effect mainly originates from frequency dependence of dielectric permittivity $\Delta \varepsilon$ since all other parameters are independent of frequency.

\section{Conclusions}

Dielectric and electrooptical measurements were carried out on the novel liquid crystal mixture with frequency tunable capabilities from $100 \mathrm{~Hz}$ to $10 \mathrm{KHz}$. The dielectric anisotropy of the proposed mixture goes to zero at higher frequencies instead of being negative, which is typical for dual frequency nematics (DFN). Characterization protocols allowing the optimum classification of different components 
of this mixture, paying attention to all relevant parameters such as anisotropic polarizability and dielectric anisotropy have been presented. The dielectric permittivity of the mixtures based on the three components depends upon the polarity of each particular group and their concentrations in the mixture. These studies can aid the development of new liquid crystals materials with carefully tailored dielectric properties.

Author Contributions: Methodology, writing and editing, N.B.; optical retardance measurement, A.K.; synthesis of the liquid crystalline single compounds and formulation of the mixture, J.H.; discussion of the results, P.K.; and supervision of the work, L.R.J.

Funding: Ministry of National Defense of Poland under grant number (GBMON/13-995/2018/WAT), Military University of Technology grant no 23-895.

Acknowledgments: The authors would like to thank Stanisław Urban for the dielectric measurements and his helpful discussions.

Conflicts of Interest: The authors declare no conflict of interest.

\section{References}

1. Dąbrowski, R.; Dziaduszek, J.; Garbat, K.; Urban, S.; Filipowicz, M.; Herman, J.; Czerwiński, M.; Harmata, P. Nematic compounds and mixtures with high negative dielectric anisotropy. Liq Cryst 2017, 44, 1534-1548.

2. Apter, B.; Efron, U.; Bahat-Treidel, A. On the fringing-field effect in liquid-crystal beam-steering devices. Appl. Opt. 2004, 43, 11-19. [CrossRef] [PubMed]

3. Cuypers, D.; De Smet, H.; Van Calster, A. Electronic compensation for fringe-field effects in VAN LCOS microdisplays. In Proceedings of the International Symposium of the Society-for-Information-Display, Campbell, CA, USA, 18-23 May 2008.

4. Daniel, V. Dielectric Relaxation; Academic Press: London, UK; New York, NY, USA, 1967; p. 203.

5. Zakharov, A.V.; Dong, R.Y. Dielectric and elastic properties of liquid crystals. Phys. Rev. E. 2001, 64, 031701. [CrossRef]

6. Kresse, H. Dielectric Behaviour of Liquid Crystals. In Advances in Liquid Crystals; Academic Press: New York, NY, USA, 1983; pp. 109-171.

7. Jadzyn, J.; Hellemans, L.; Czechowski, G.; Legrand, C.; Douali, R. Dielectric and viscous properties of 6CHBT in the isotropic and nematic phases. Liq Cryst 2000, 27, 613-619. [CrossRef]

8. Maier, W.; Meier, G. Anisotrope dk-dispersion im radiofrequenzgebiet bei homogen geordneten kristallinen flussigkeiten. Z. Naturforsch 1961, 16, 1200-1205.

9. Toriyama, K.; Sugimori, S.; Moriya, K.; Dunmur, D.A.; Hanson, R. Dielectric study of dipole-dipole interactions in anisotropic solutions. J. Phys. Chem 1996, 100, 307-315. [CrossRef]

10. Dabrowski, R.; Celiński, M.; Chojnowska, O.; Kula, P.; Dziaduszek, J.; Urban, U. Compounds with low relaxation frequency and dual frequency mixtures useful for active matrix addressing. Liq Cryst 2013, 40, 1339-1353.

11. Perkowski, P.; Mrukiewicz, M.; Garbat, K.; Laska, M.; Chodorow, U.; Piecek, W.; Dabrowski, R.; Parka, J. Precise dielectric spectroscopy of a dual-frequency nematic mixture over a broad temperature range. Liq Cryst 2012, 39, 1237-1242. [CrossRef]

12. Ibragimov, T.D.; Bayramov, G.M. Application of dual-frequency liquid crystal for tunable selective filtration of infrared radiation. Optik 2013, 124, 6666-6668. [CrossRef]

13. Song, Q. Fast Response Dual Frequency Liquid Crystal Materials. Ph.D. Thesis, University of Central Florida, Orlando, FL, USA, 2010.

14. Song, Q.; Jiao, M.; Ge, Z.; Xianyu, H.; Gauza, S.; Wu, S.T. High birefringence and low crossover frequency dual frequency liquid crystals. Mol. Cryst. Liq. Cryst 2008, 488, 179-189. [CrossRef]

15. Xianyu, H.; Gauza, S.; Wu, S.T. Sub-millisecond response phase modulator using a low crossover frequency dual-frequency liquid crystal. Liq Cryst 2008, 35, 1409-1413. [CrossRef]

16. Kula, P.; Aptacy, A.; Herman, J.; Wojciak, W.; Urban, S. The synthesis and properties of fluoro-substituted analogues of 4-butyl-4'-[(4-butylphenyl)ethynyl]biphenyls. Liq Cryst 2013, 40, 482-491. [CrossRef]

17. Bailey, J.; Kaur, S.; Morgan, P.B.; Gleeson, H.F.; Clamp, J.H.; Jones, J.C. Design considerations for liquid crystal contact lenses. J. Phys. D. Appl. Phys. 2017, 50, 485401. [CrossRef] 
18. Oton, E.; Morawiak, P.; Mazur, R.; Quintana, X.; Geday, M.A.; Oton, J.M.; Piecek, W. Diffractive and Refractive Liquid Crystal Devices Based on Multilayer Matrices. J. Light. Technol. 2019, 37, 2086-2093. [CrossRef]

19. Kotova, S.P.; Patlan, V.V.; Samagin, S.A.; Zayakin, O.A. Wavefront formation using modal liquid-crystal correctors. Phys. Wave Phenom. 2010, 18, 96-104. [CrossRef]

20. Algorri, J.F.; Urruchi del Pozo, V.; Sanchez-Pena, J.M.; Oton, J.M. An Autostereoscopic Device for Mobile Applications Based on a Liquid Crystal Microlens Array and an OLED Display. J. Disp. Technol. 2014, 10, 713-720. [CrossRef]

21. Algorri, J.F.; Urruchi, V.; Bennis, N.; Sánchez-Pena, J.M. Cylindrical liquid crystal microlens array with rotary axis and tunable capability. IEEE Electron Device Lett. 2015, 36, 582-584. [CrossRef]

22. Algorri, J.F.; Urruchi, V.; Bennis, N.; Morawiak, P.; Sanchez-Pena, J.M.; Oton, J.M. Integral Imaging Capture System with Tunable Field of View Based on Liquid Crystal Microlenses. IEEE Photonics Technol. Lett. 2016, 28, 1854-1857. [CrossRef]

23. Algorri, J.F.; Urruchi, V.; Bennis, N.; Sánchez-Pena, J.M.; Otón, J.M. Tunable liquid crystal cylindrical micro-optical array for aberration compensation. Opt. Express 2015, 23, 13899-13915. [CrossRef] [PubMed]

24. Algorri, J.F.; Urruchi, V.; Bennis, N.; Sánchez-Pena, J.M. Modal liquid crystal microaxicon array. Opt. Lett. 2014, 39, 3476-3479. [CrossRef]

25. Algorri, J.F.; Love, G.D.; Urruchi, V. Modal liquid crystal array of optical elements. Opt. Express 2013, 21, $24809-24818$. [CrossRef]

26. Urruchi, V.; Algorri, J.F.; Marcos, C.; Sánchez-Pena, J.M. Electrical modeling and characterization of voltage gradient in liquid crystal microlenses. Rev. Sci. Instrum 2013, 84, 116105. [CrossRef]

27. Algorri, J.F.; Bennis, N.; Urruchi, V.; Morawiak, P.; Sánchez-Pena, J.M.; Jaroszewicz, L.R. Tunable liquid crystal multifocal microlens array. Sci. Rep. 2017, 7, 17318. [CrossRef]

28. Algorri, J.F.; Urruchi, V.; Bennis, N.; Morawiak, P.; Sánchez-Pena, J.M.; Otón, J.M. Liquid crystal spherical microlens array with high fill factor and optical power. Opt. Express 2017, 25, 605. [CrossRef]

29. Algorri, J.F.; Bennis, N.; Herman, J.; Kula, P.; Urruchi, V.; Sánchez-Pena, J.M. Low aberration and fast switching microlenses based on a novel liquid crystal mixture. Opt. Express 2017, 25, 14795. [CrossRef]

30. Algorri, J.F.; Urruchi, V.; Garcia-Camara, B.; Sanchez-Pena, J.M. Generation of Optical Vortices by an Ideal Liquid Crystal Spiral Phase Plate. IEEE Electron Device Lett 2014, 35, 856-858. [CrossRef]

31. Algorri, J.; Urruchi, V.; García-Cámara, B.; Sánchez-Pena, J. Liquid Crystal Lensacons, Logarithmic and Linear Axicons. Materials 2014, 7, 2593-2604. [CrossRef]

32. Kula, P.; Dziaduszek, J.; Herman, J.; Dabrowski, R. Invited Paper: Highly Birefringent Nematic Liquid Crystals and Mixtures. SID Symposium Digest Technical Papers 2014, 45, 100-103. [CrossRef]

33. Dabrowski, R.; Kula, P.; Herman, J. High Birefringence Liquid Crystals. Liq Cryst 2013, 3, 443-482. [CrossRef]

34. Weglowska, D.; Kula, P.; Herman, J. High birefringence bistolane liquid crystals: synthesis and properties. RSC Adv. 2016, 6, 403-408. [CrossRef]

35. Herman, J.; Kula, P. Design of new super-high birefringent isothiocyanato bistolanes-synthesis and properties. Liq Cryst 2017, 44, 1462-1467. [CrossRef]

36. Ziobro, D.; Kula, P.; Dziaduszek, J.; Filipowicz, M.; Dabrowski, R.; Parka, J.; Czub, J.; Urban, S.; Wu, S.T. Mesomorphic and dielectric properties of esters useful for formulation of nematic mixtures for dual frequency addressing system. Opto-Elect Rev 2009, 17, 16-19. [CrossRef]

37. Wrobel, S.; Brodzik, M.; Dabrowski, R.; Gestblom, B.; Haase, W.; Hiller, S. Relaxation phenomena in the nematic, nematic reentrant and smectic A phases studied by dielectric spectroscopy. Mol. Cryst. Liq. Cryst 1997, 302, 223-228. [CrossRef]

(C) 2019 by the authors. Licensee MDPI, Basel, Switzerland. This article is an open access article distributed under the terms and conditions of the Creative Commons Attribution (CC BY) license (http://creativecommons.org/licenses/by/4.0/). 